\title{
Verification of the Solar Dynamics Observatory High Gain Antenna Pointing Algorithm Using Flight Data
}

\author{
Kristin L. Bourkland* and Kuo-Chia (Alice) $\mathrm{Liu}^{\dagger}$ \\ NASA Goddard Space Flight Center, Greenbelt, MD, 20771, US
}

\begin{abstract}
$\mathrm{T}$ HE Solar Dynamics Observatory (SDO) is a NASA spacecraft designed to study the Sun. It was launched on February 11, 2010 into a geosynchronous orbit, and uses a suite of attitude sensors and actuators to finely point the spacecraft at the Sun. SDO has three science instruments: the Atmospheric Imaging Assembly (AIA), the Helioseismic and Magnetic Imager (HMI), and the Extreme Ultraviolet Variability Experiment (EVE). SDO uses two High Gain Antennas (HGAs) to send science data to a dedicated ground station in White Sands, New Mexico. In order to meet the science data capture budget, the HGAs must be able to transmit data to the ground for a very large percentage of the time.

Each HGA is a dual-axis antenna driven by stepper motors. Both antennas transmit data at all times, but only a single antenna is required in order to meet the transmission rate requirement. For portions of the year, one antenna or the other has an unobstructed view of the White Sands ground station. During other periods, however, the view from both antennas to the Earth is blocked for different portions of the day. During these times of blockage, the two HGAs take turns pointing to White Sands, with the other antenna pointing out to space. The HGAs handover White Sands transmission responsibilities to the unblocked antenna. There are two handover seasons per year, each lasting about 72 days, where the antennas hand off control every twelve hours. The non-tracking antenna slews back to the ground station by following a ground commanded trajectory and arrives approximately 5 minutes before the formerly tracking antenna slews away to point out into space.

The SDO Attitude Control System (ACS) runs at $5 \mathrm{~Hz}$, and the HGA Gimbal Control Electronics (GCE) run at $200 \mathrm{~Hz}$. There are 40 opportunities for the gimbals to step each ACS cycle, with a hardware limitation of no more than one step every three GCE cycles. The ACS calculates the desired gimbal motion for tracking the ground station or for slewing, and sends the command to the GCE at $5 \mathrm{~Hz}$. This command contains the number of gimbals steps for that ACS cycle, the direction of motion, the spacing of the steps, and the delay before taking the first step.

The AIA and HMI instruments are sensitive to spacecraft jitter. Pre-flight analysis showed that jitter from the motion of the HGAs was a cause of concern. Three jitter mitigation techniques were developed to overcome the effects of jitter from different sources. The first method is the random step delay, which avoids gimbal steps hitting a cadence on a jitter-critical mode by pseudo-randomly delaying the first gimbal step in an ACS cycle. The second method of jitter mitigation is stagger stepping, which forbids the two antennas from taking steps during the same ACS cycle in order to avoid constructively adding jitter from two antennas. The third method is the inclusion of an instrument No Step Request (NSR), which allows the instruments to request a stoppage in gimbal stepping during the times when they are taking images.

During the commissioning phase of the mission, a jitter test was performed onboard the spacecraft. Various sources of jitter, such as the reaction wheels, the High Gain Antenna motors, and the motion of the instrument filter wheels, were examined to determine the level of their effect on the instruments. During the HGA portion of the test, the jitter amplitudes from the single step of a gimbal were examined, as well as the amplitudes due to the execution of various gimbal rates. These jitter levels are compared with the gimbal jitter allocations for each instrument.

Additionally, the jitter test provided insight into a readback delay that exists with the GCE. Pre-flight analysis suggested that gimbal steps scheduled to occur during the later portion of an ACS cycle would not be read during that cycle, resulting in a delay in the telemetered current gimbal position. Flight data from the jitter test confirmed this expectation. Analysis is presented that shows the readback delay does not have a negative impact on gimbal control.
\end{abstract}

*ACS Analyist, Attitude Control Systems Engineering Branch/Code 591

${ }^{\dagger}$ ACS Analyist, Attitude Control Systems Engineering Branch/Code 591, AIAA Lifetime Member 
The decision was made to consider implementing two of the jitter mitigation techniques on board the spacecraft: stagger stepping and the NSR. Flight data from two sets of handovers, one set without jitter mitigation and the other with mitigation enabled, were examined. The trajectory of the predicted handover was compared with the measured trajectory for the two cases, showing that tracking was not negatively impacted with the addition of the jitter mitigation techniques. Additionally, the individual gimbal steps were examined, and it was confirmed that the stagger stepping and NSRs worked as designed.

An Image Quality Test was performed to determine the amount of cumulative jitter from the reaction wheels, HGAs, and instruments during various combinations of typical operations. In this paper, the flight results are examined from a test where the HGAs are following the path of a nominal handover with stagger stepping on and HMI NSRs enabled. In this case, the reaction wheels are moving at low speed and the instruments are taking pictures in their standard sequence. The flight data shows the level of jitter that the instruments see when their shutters are open. The HGA-induced jitter is well within the jitter requirement when the stagger step and NSR mitigation options are enabled.

The SDO HGA pointing algorithm was designed to achieve nominal antenna pointing at the ground station, perform slews during handover season, and provide three HGA-induced jitter mitigation options without compromising pointing objectives. During the commissioning phase, flight data sets were collected to verify the HGA pointing algorithm and demonstrate its jitter mitigation capabilities. 